REVISTA

\title{
Currículo de Autores
}

\section{Nelson Bautista}

PDVSA, Maracaibo-Venezuela

Magíster en Gerencia de Proyectos Industriales de la Universidad Dr. Rafael Belloso Chacín; Ingeniero en Informática de la Universidad Dr. Rafael Belloso Chacín. Cuenta con 18 años de experiencia en el área de proyectos informáticos, áreas de seguimiento, contratación, evaluación económica, ingeniería, diseño de sistemas informáticos, control de proyectos, supervisión y control de obras.

\section{Patricia Castillo Jiménez}

Universidad del Zulia, Maracaibo - Venezuela

Magíster en Gerencia de Empresas con mención en Operaciones de la Universidad del Zulia; Ingeniero Industrial. De la Universidad Alonso de Ojeda. Consultor técnico para: elaboración de planes de calidad y seguridad industrial; preparación para certificación bajo normas ISO 9001 , OHSAS 18001, ISO 14001, HACCP, NCH 2728 (OTEC). Analista de seguridad industrial e higiene ocupacional.

\section{Micaela Gallardo Montevilla}

Instituto de Investigación, Consultoría y Servicios Turísticos, La Paz - Bolivia

Profesional en Turismo, ejerce como guía de sitio, asistente y counter turístico. Con experiencia en elaboración de proyectos. Miembro del equipo de trabajo del Instituto de Investigación de Turismo IICSTUR. Auxiliar de docencia en la Universidad Mayor de San Andrés

\section{Denys García de Mujica}

Universidad Dr. Rafael Belloso Chacín, Maracaibo - Venezuela

Doctora en Ciencias Gerenciales de la Universidad Dr. Rafael Belloso Chacín; Magíster en Planificación y Gerencia de Ciencia y Tecnología de la Universidad del Zulia; Magíster en Dirección Estratégica de Personal 2.0 de la Universidad de Alcalá - España; Ingeniera en Industria de la Universidad del Zulia. Cuenta con 17 años de experiencia docente y 33 en el área de ingeniería industrial y en el área gerencial de recursos humanos, planificación y control de proyectos y estimación de costo. 
REVISTA

\section{Currículo de Autores}

\section{Paola López}

Universidad del Zulia, Maracaibo - Venezuela

Ingeniera Civil de la Universidad del Zulia. Investigadora estudiantil en el Laboratorio de Investigaciones Ambientales del Núcleo Costa Oriental del Lago de LUZ. Miembro del equipo técnico de ingeniera civil en la empresa Desarrollos Urbanos S.A. de Venezuela.

\section{Marehilen Lugo}

Universidad del Zulia, Maracaibo - Venezuela

Ingeniera Civil de la Universidad del Zulia. Investigadora estudiantil en el Laboratorio de Investigaciones Ambientales del Núcleo Costa Oriental del Lago de LUZ.

\section{Marle Cecilia Martínez Ramírez}

Universidad del Zulia, Maracaibo - Venezuela

Doctor of Science in Systems Engineering; Magíster en Informática Educativa; Ingeniero en Computación. Docente-investigadora a nivel de pregrado y postgrado. Profesora titular con dedicación exclusiva en la Universidad del Zulia. Publicaciones en el área de Tecnologías de la información y comunicación, sistemas de información, gestión del conocimiento, gestión tecnológica, indicadores de gestión, diseño instruccional y aprendizaje virtual.

\section{Iván Mendoza}

Universidad del Zulia, Maracaibo - Venezuela

Doctor en Ciencias de la Educación; Magister en Ciencias Ambientales; Licenciado en Educación, Mención Biología y Química. Profesor Emérito de la Universidad del Zulia. Investigador en el área ambiental y educativa. Orden Dr. Jesús Enrique Lossada en Primera y Segunda Clase. 
REVISTA

\section{Currículo de Autores}

\section{Adryd Mujica}

ROSSETI, Córdoba-Argentina

Magíster en Gerencia de Proyectos Industriales de la Universidad Dr. Rafael Belloso Chacín; Ingeniero en Industria de la Universidad Dr. Rafael Belloso Chacín. Cuenta con 7 años de experiencia en el área de proyectos Industriales, analista de planificación y control de proyectos. Experto en actividades correspondientes a la planificación, ejecución, control y planes de contingencia de los proyectos. Trabajó en empresas dedicadas al desarrollo de proyectos de construcción en Venezuela como Dragasur, GEO Servicios Corporativos, C.A. y en Argentina en ROSSETI en el desarrollo de proyectos Eólicos.

\section{Susan Pantelís}

Universidad Mayor de San Andrés, La Paz - Bolivia

Profesional en Turismo, investigadora activa en temas relacionados con el turismo comunitario en Bolivia, comunicación, diplomacia y operación de servicios turísticos. Actualmente cursa la Maestría en Desarrollo Turístico Sustentable en la Universidad Mayor de San Andrés, La Paz - Bolivia.

\section{Camila Elizabeth Rojas Gareca}

Universidad Mayor de San Andrés, La Paz - Bolivia

Profesional en Turismo, investigadora activa en temas relacionados con el turismo comunitario en Bolivia.

\section{Amed Said Uceda Mercado}

Instituto Técnico CEC, Oruro-Bolivia

Profesional en Turismo. Docente de turismo, inglés como segunda lengua y aplicación del inglés en el ámbito turístico. Fue parte de la consultora de turismo rural A-Tec en el diseño de su aplicación Android. Actualmente cursa una Maestría en Desarrollo Turístico Sustentable. 